\title{
HYDROGEN MONITORING REQUIREMENTS IN THE GLOBAL TECHNICAL REGULATION ON HYDROGEN AND FUEL CELL VEHICLES
}

\author{
W. Buttner ${ }^{1}$, C. Rivkin ${ }^{1}$, R. Burgess ${ }^{1}$, K. Hartmann ${ }^{1}$, I. Bloomfield ${ }^{1}$, M. Bubar ${ }^{1}$, M. Post ${ }^{1}$, \\ L. Boon-Brett ${ }^{2}$, E. Weidner ${ }^{2}$, and P. Moretto ${ }^{2}$ \\ ${ }^{1}$ National Renewable Energy Laboratory, Hydrogen \& Fuel Cell Systems Engineering \\ Group, Hydrogen Safety Codes and Standards Group, Golden, CO (USA). \\ (william.buttner@nrel.gov) \\ ${ }^{2}$ European Commission, DG Joint Research Centre, Institute for Energy and Transport - \\ Energy Conversion and Storage Technologies Unit, P.O. Box 2, 1755 ZG Petten, The \\ Netherlands. \\ (eveline.weidner@.ec.europa.eu)
}

\begin{abstract}
The United Nations Economic Commission for Europe Global Technical Regulation (GTR) Number 13 (Global Technical Regulation on Hydrogen and Fuel Cell Vehicles) is the defining document regulating safety requirements in hydrogen vehicles, and in particular, fuel cell electric vehicles (FCEVs). GTR Number 13 has been formally adopted and will serve as the basis for the national regulatory standards for FCEV safety in North America (led by the United States), Japan, Korea, and the European Union. The GTR defines safety requirements for these vehicles, including specifications on the allowable hydrogen levels in vehicle enclosures during in-use and post-crash conditions and on the allowable hydrogen emissions levels in vehicle exhaust during certain modes of normal operation. However, in order to be incorporated into national regulations, that is, to be legally binding, methods to verify compliance with the specific requirements must exist. In a collaborative program, the Sensor Laboratories at the National Renewable Energy Laboratory in the United States and the Joint Research Centre, Institute for Energy and Transport in the Netherlands have been evaluating and developing analytical methods that can be used to verify compliance with the hydrogen release requirements as specified in the GTR.
\end{abstract}

Key Words: Global Technical Regulation; hydrogen sensors; fuel cell vehicle; safety

\section{INTRODUCTION}

The U.S. Department of Energy (DOE) Fuel Cell Technologies Office has taken the lead to support the development and deployment of hydrogen as an alternative energy source in the United States [1]. It supports DOE's mission to ensure the United States' security and prosperity by addressing energy and environmental challenges through transformative science and technology solutions [2]. Similarly, the European Commission identified the potential of hydrogen and fuel cells in the 2011 Technologies Map of the European Strategic Energy Technology Plan [3]. Hydrogen infrastructure and vehicles must be developed safely if hydrogen is to be used successfully as a fuel. One element of a hydrogen safety system is the use of sensors to detect and monitor unexpected hydrogen releases. Accordingly, sensor test facilities were independently established by the European Joint Research Centre (JRC) at the Institute for Energy and Transport [4] and by DOE at the National Renewable Energy Laboratory (NREL) [5] to ensure that hydrogen sensors are available to meet the needs of the hydrogen infrastructure and to educate the hydrogen community on the proper use of hydrogen sensors. These laboratories have ongoing collaborative sensor research programs formalized by a Memorandum of Agreement, and more recently under an agreement between DOE Fuel Cell Technologies Office and the Fuel Cell and Hydrogen Joint Undertaking (FCH-JU) of the European Union, in which it was agreed to collaborate on hydrogen sensor research. This agreement was the first formal international collaboration with common objectives between the hydrogen programs within the United States DOE and FCH-JU. The goals were to identify 
gaps in current sensor technologies and to identify pathways to make available effective, cost efficient sensors. The EU activity was performed under the auspices of H2Sense [6], a FCH-JU-funded consortium of European sensor manufacturers and research laboratories. The U.S. activity was headed by the NREL Sensor Test Laboratory.

\subsection{Global Technical Regulation Number 13}

United Nations Economic Commission for Europe Global Technical Regulation (GTR) Number 13 (Global Technical Regulation on Hydrogen and Fuel Cell Vehicles) [7] is the defining document regulating the safety requirements for light-duty hydrogen vehicles, and in particular fuel cell electric vehicles (FCEV). GTR Number 13 has been formally adopted and thus is to serve as the basis for the national regulations for FCEV safety in North America (led by the United States), Japan, Korea, and the European Union. Vehicle safety regulations are implemented and enforced by national authorities. A goal of the GTR is to provide a framework to internationally harmonize FCEV safety requirements to facilitate hydrogen vehicle market development and trade. Accordingly, national authorities overseeing development and enforcement of vehicle regulations in their respective jurisdictions shall endeavor to harmonize their national regulations with the GTR. Within the United States, the national authority for vehicle safety is the U.S. Department of Transportation (DOT), National Highway Traffic Safety Administration (NHTSA) and the prevailing regulatory code is the Federal Motor Vehicle Safety Standard. Under the terms of the agreement for the implementation of the GTR, national authorities overseeing vehicle safety regulations are required to make a "good-faith" effort to harmonize their respective regulations with the GTR by either complying with the terms as they currently exist or by appealing to change specific terms or requirements within the GTR deemed unacceptable. Therefore, compliance with the GTR is not absolutely mandatory, and national regulations are able to deviate from the GTR requirements provided an attempt for harmonization was made by the respective national authority. An opportunity to formally recommend revisions to the current version of the regulation is tentatively scheduled for late 2016 .

The GTR covers electrical, mechanical, pressure, and other safety requirements for FCEVs. Included within the GTR are safety requirements on allowable hydrogen emission levels in vehicle enclosures during in-use and post-crash test conditions and on the allowable hydrogen content in vehicle exhaust during certain modes of normal operation. However, in order to be incorporated into national regulations, that is, to be binding, methods to verify compliance with the specific requirements must exist. In a collaborative program, the sensor laboratories at NREL in the United States and the JRC in the Netherlands have been developing analytical methods that can be used to verify compliance with the hydrogen emission requirement as specified in the GTR. There are two specific requirements defining allowable hydrogen releases specified in the GTR. These are presented in in Section 5 (Performance Requirements). The specific hydrogen release requirements are:

Section 5.2.1.3.2 - Vehicle Exhaust System

At the vehicle exhaust system's point of discharge, the hydrogen concentration level shall:

(a) Not exceed 4 percent average by volume during any moving three-second time interval during normal operation including start-up and shutdown;

(b) And not exceed 8 percent at any time.

Section 5.2.2.2. Concentration limit in enclosed spaces (Post Crash Test Integrity)

Hydrogen gas leakage shall not result in a hydrogen concentration in the air greater than $3 \pm 1.0$ percent by volume in the passenger, luggage and cargo compartments.

The NREL and JRC sensor laboratories have ongoing activities to address both released hydrogen detection requirements as listed in the GTR and summarized above. This information has been shared 
with the DOT to provide DOT with tools to verify compliance with the GTR requirement or to supply normative data to support recommendations to modify the current requirements. The results of this research are presented below.

\section{VEHICLE CRASH TEST REQUIREMENTS}

\subsection{Sensors and Methods for Monitoring Hydrogen/Helium in FCEV Crash Tests}

The NREL and JRC sensor laboratories completed two studies pertaining to the hydrogen monitoring requirements specified in Section 5.2 of the GTR for fuel system integrity following vehicle crash tests. In the first study, NREL had the opportunity to instrument a demonstration FCEV with hydrogen sensors in an actual crash test. This was performed in cooperation with the DOT NHTSA. The goal of the project was to identify a sensor or sensor method that could respond to either hydrogen or helium releases, identify a technology that could survive the impacts of vehicle crash tests and still meet the metrological requirements for verification of the FCEV fuel system integrity as per the GTR requirements, and to develop a method for on-board monitoring of the sensor either in real time or extractable for post-crash test analysis. The results of this study are briefly summarized in the next section, and a comprehensive summary was published as an NREL report [8]. All project requirements were successfully completed.

A second study by NREL and the JRC assessed the GTR recommendation that hydrogen determinations can be made by measuring the displacement (decrease) of oxygen in vehicle compartments [9].

\subsection{Sensors for Verification of Fuel System Integrity Following Crash Tests}

Although there are a variety of hydrogen sensor platforms that can respond to hydrogen, most will not respond to helium. This is a concern in that the GTR allows helium to be used as a surrogate for hydrogen in vehicle crash tests. In the demonstration crash test, the vehicle fuel system was pressurized with helium [9]. One specific requirement for post-crash test conditions is that hydrogen shall not build up to a concentration greater than $4 \mathrm{vol} \%$ in any vehicle compartment (e.g., passenger compartment, trunk, fuel cell enclosure) within 1 hour following crash test impact or, if helium is used as a surrogate, no greater than 3 vol\%.

One sensor platform that is sensitive to hydrogen as well as helium is the thermal conductivity (TC) sensor, which is commercially available from numerous suppliers, including models configured in physically robust packages. Although the TC sensor shows different sensitivity to hydrogen versus helium, as shown in Figure 1, this is not a problem because in a crash test it will be known whether helium or hydrogen is used. Of course, hydrogen will always be used in normal vehicle operation. For the study reported in [9], the vehicle fuel system was pressurized with helium.

Five units of a commercial TC sensor configured in a physically robust package were obtained for deployment in vehicle crash tests. The identified sensor proved to be very robust and compatible with the impacts and shocks associated with crash test. Indeed, individual units of the selected sensor were subjected up to five vehicle crash tests without a single incident of device failure or even alteration of measurement accuracy as confirmed by pre- and post-crash test laboratory calibrations.

In collaboration with engineers at Karco Engineering [10], an engineering firm certified to perform vehicle crash tests, a mechanical fixture was designed for securing the TC sensor at the desired locations within the vehicle. The fixture was demonstrated and used in FCEV crash tests. Two FCEVs were available and were subjected to the side impact test and to the rear impact test. Although the sensors were mounted in the vehicle, monitoring was performed external to the vehicle via an umbilical that interfaced 
the sensor output to a remote data acquisition system. ${ }^{1}$ The sensors successfully monitored the integrity of the fuel system within the vehicle passenger compartment and underneath the vehicle near the fuel tanks. Although small helium releases were detected, these were deemed to be primarily an artifact of the crashtest procedure, and the vehicle was shown to be in compliance with the GTR. The main conclusions from this study included:

- $\quad$ TC sensors are compatible for both hydrogen and helium measurements.

- Appropriately packaged TC sensors are compatible with vehicle crash tests and have the metrological performance specifications as required in the GTR.

- Access to the sensor output in real time during the vehicle crash test and the subsequent 1-hour hold period following impact is recommended (via, for example, telemetry) in the event unsafe levels of hydrogen are building up in the vehicle compartment.
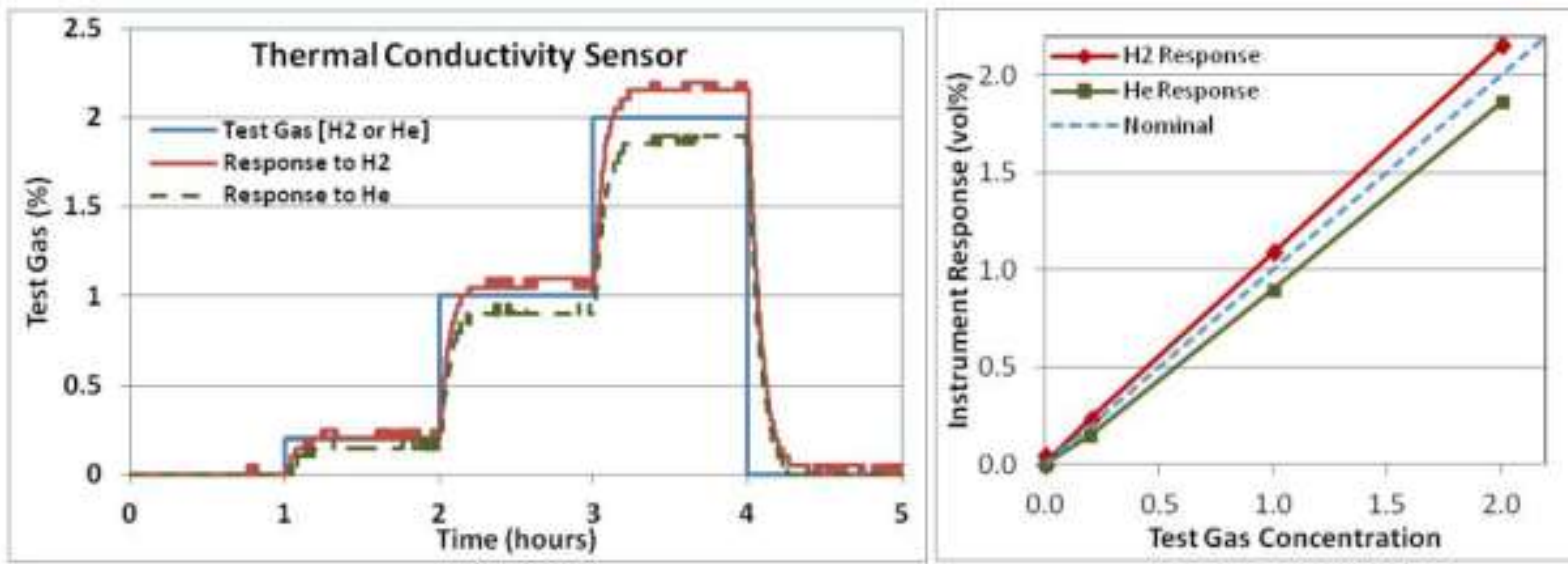

Figure 1: (Left) Response of a commercial TC sensor to $0.2,1.0$, and 2.0 vol $\% \mathrm{H}_{2}$ and He. (Right) The corresponding final indication of the sensor to hydrogen and helium. The sensor was factory calibrated to output a response equivalent to vol\% $\mathrm{H}_{2}$. Details in [8].

\subsection{Measurement of Oxygen Displacement to Quantify Hydrogen Releases}

The GTR also has a recommendation that following crash tests, hydrogen releases could be measured via reduction in oxygen (due to displacement of air by leaking hydrogen/helium). The use of oxygen sensors to quantify hydrogen releases has been used by several groups, especially for modelling studies of hydrogen releases (e.g., [11, 12]). The viability of this approach was critiqued by the NREL and JRC sensor laboratories, and the results have also been previously reported $[9,13]$. Using oxygen sensors to measure oxygen displacement to quantify hydrogen has several apparent advantages. These include the low cost and ease of use of oxygen sensors and the fact that displacement measurements should be compatible with either hydrogen or helium and thus would seem ideal for modeling studies using helium as a hydrogen surrogate. After a thorough investigation by the NREL and JRC sensor laboratories, it was shown that this approach should be avoided. The fundamental electronic signal noise of oxygen sensors renders this method of hydrogen detection inadequate for safety applications. Normal drift in the oxygen sensor could lead to hydrogen false positive alarms, or even worse, false negative alarms.

The oxygen sensor is also sensitive to the partial pressure of oxygen $\left(\mathrm{P}_{\mathrm{O} 2}\right)$ and not vol\% $\mathrm{O}_{2}$, and thus would not respond to hydrogen releases in a closed environment, such as for example a sealed vehicle compartment. In a closed environment, a hydrogen release may lower the vol $\% \mathrm{O}_{2}$, but would not affect $\mathrm{P}_{\mathrm{O} 2}$. It was explicitly shown that no change in the oxygen sensor signal was observed even when up to 20 vol\% He was added to a sealed air chamber [9] whereas a TC sensor installed in the same test chamber responded to the helium. Furthermore, the oxygen sensor would not accurately respond when the release

\footnotetext{
${ }^{1}$ Data acquisition systems compatible with both crash tests and the sensor output exist that could operate on-board the vehicle, but these were not available in time for the actual crash test.
} 
induced a pressure gradient (e.g., as would be the case when a release into a room induces remote venting). The oxygen sensor signal is also very much dependent upon fluctuations in environmental parameters, especially temperature and pressure. Although pressure variations are typically small, temperature changes can be significant, especially, for example, in a closed vehicle parked in the sun prior to and during the crash test. Although the temperature dependence of many oxygen sensors is minimized by the incorporation of a compensating temperature sensor (typically a thermistor or resistance temperature device--RTD), a measurable temperature dependence of the sensor signal is still observed [9]. The impact of temperature dependence can be to some extent corrected, typically using steady-state measurements at various fixed temperatures to develop temperature-dependent correction factors. However, the effect of temperature transients can be complex. This is due in part to different thermal inertia associated with the oxygen sensing element and the compensating thermistor or RTD that is often integrated into the oxygen sensor. An example of sensor responses from a commercial oxygen sensor during temperature changes is shown in Figure 2. The sensor response is monitored for a constant oxygen concentration, and it is shown that change in its response due to temperature does not exactly follow the temperature change within the test chamber; in fact, the sensor response shows a well-defined minimum during monotonic cooling of the test chamber from $40^{\circ} \mathrm{C}$ to $25^{\circ} \mathrm{C}$. The actual behavior of a particular oxygen sensor model during temperature variation is very much dependent upon its specific design and different behavior can be observed with a different sensor design.

Finally, if relying on displacement to quantify a specific diluent, it must be kept in mind that the oxygen sensor readout cannot differentiate between different diluents. This makes it easy to substitute helium for hydrogen in crash tests. However, in a vehicle crash test, the displacement gas may come from the vehicle fuel system or from other sources, such as the venting of air bags. It was concluded that the use of oxygen sensors to (indirectly) quantify hydrogen or helium releases should be avoided $[9,13]$. Furthermore, it is not necessary since, as was shown in the vehicle crash test study described in [9], the TC sensor can be used to measure either hydrogen or helium and can do so with better measuring range, detection limits, and accuracy than the oxygen sensor.

It was strongly recommended to DOT, the U.S. regulatory agency for vehicle safety and the U.S. representative on the GTR, that the text endorsing oxygen displacement as a means for quantifying

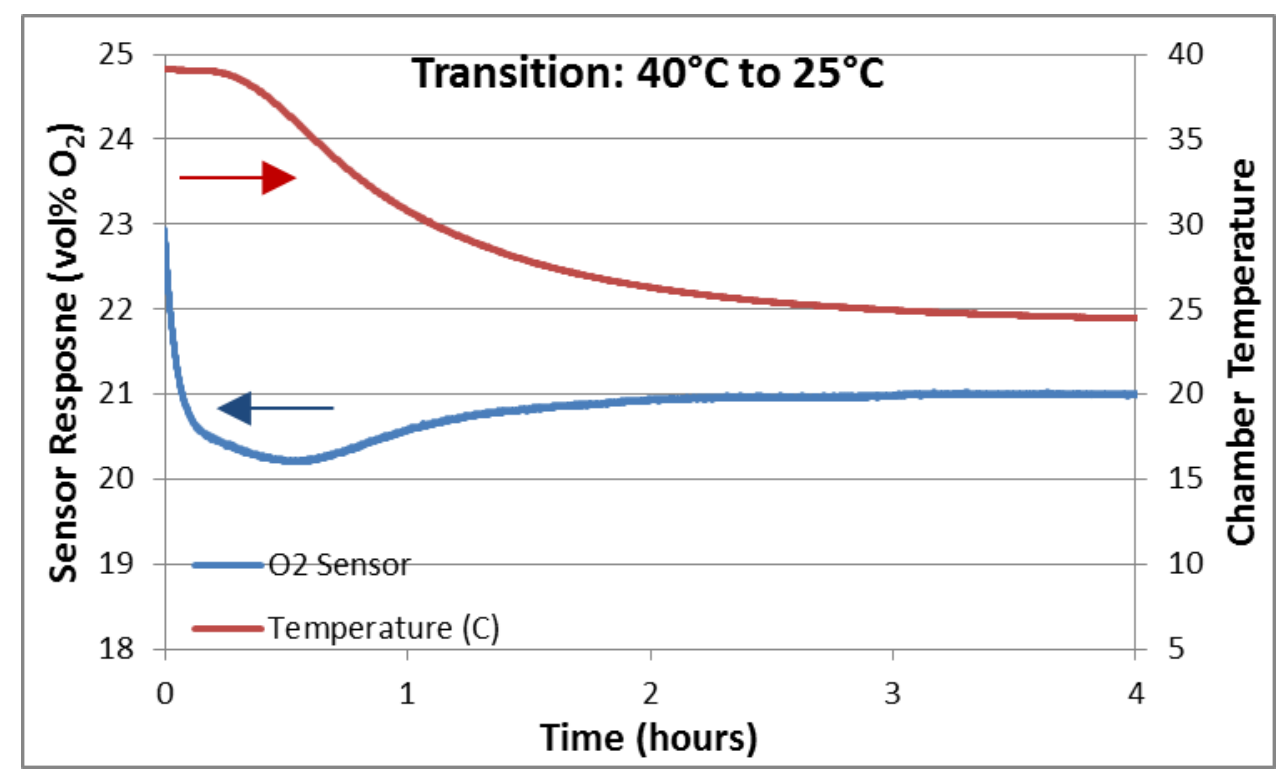

Figure 2: Response of a commercial oxygen sensor in air to transient temperature changes from $40^{\circ} \mathrm{C}$ to $25^{\circ} \mathrm{C}$. Although a well-defined steady-state oxygen sensor signal was achieved once the chamber temperature stabilized, the sensor response to transient temperature changes was more complicated. 
hydrogen releases into vehicle compartments be removed from the GTR.

\section{ANALYZER FOR THE VERIFICATION OF FCEV TAIL PIPE EMISSION}

A second hydrogen release scenario addressed in GTR Number 13 strives to ensure that the exhaust from an FCEV is non-hazardous. The GTR imposes restrictions on allowable hydrogen releases in normal operation. A performance-based test was developed to verify that the exhaust is non-ignitable. The test requires that the average hydrogen concentration of the exhaust gas remains below the commonly accepted lower flammable limit of $4 \mathrm{vol} \%$. The average concentration is to be calculated as a 3 -second rolling average so as to accommodate extremely short, non-hazardous transients that cannot exceed 8 vol\% $\mathrm{H}_{2}$. This value was deemed acceptable based on an investigation quoted in GTR Number 13 that demonstrated that hydrogen flame propagation will not occur below 8 vol\% $\mathrm{H}_{2}$ [14]. To verify compliance with the discharge requirement, a hydrogen detector with a response time of $300 \mathrm{~ms}$ was recommended so as to detect hydrogen pulses up to 8 vol\% of one second duration. The criterion defining the response time was specified as the time to reach $90 \%$ of the sensor final indication.

The availability of hydrogen sensors with response times less than 1 second was identified by DOE as a critical metric and a performance gap [15]. To date this metric remains an elusive target. No sensor has been shown to have a 1-second response time using a validated response time apparatus and protocols [16]. Even as recently as 2013, the fastest measured hydrogen sensor response time was 4 seconds [13]. However, sensors with improved performance metrics, including response times, are continually being developed, often through miniaturization and improved mode of operation. Recently, a microfabricated thermal conductivity hydrogen sensor was identified as potentially meeting the response time requirements, if operated in a dynamic flowing gas stream. An image of the sensor is shown in Figure 3. This sensor is a thermal conductivity sensor (model XEN-TCG3880, Xensor Corporation, the Netherlands $)^{2}$ and was identified through collaboration of the NREL sensor test laboratory with the FCHJU-funded project H2Sense [6]. Although not yet independently verified, the manufacturer reported a response time of less than $250 \mathrm{~ms}$. Test results described below substantiate the fast response time. The sensor was made available in the standard package and in "fast" and "ultra-fast" configurations.

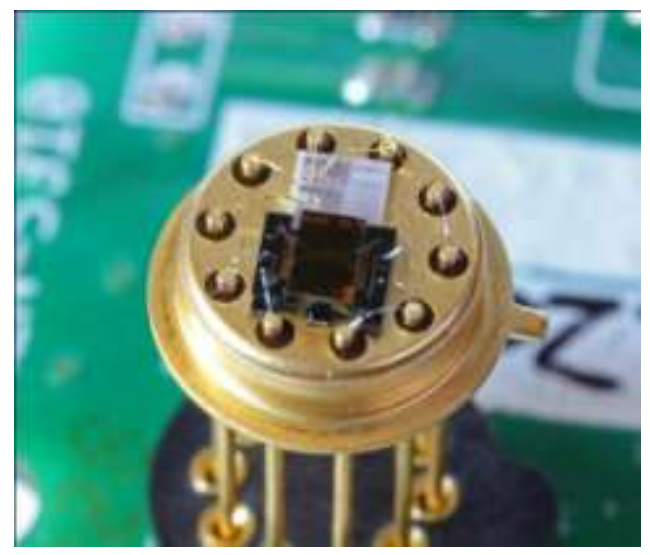

Figure 3: The "fast configuration" of the Xensor TC Sensor.

Photo used by permission [http://www.xensor.com/]

Sensor models for the standard, fast, and ultra-fast configurations were obtained, along with software for monitoring and logging of the sensor signal. At the same time, a means of generating gas pulses having well-defined compositions and durations was developed. This was achieved by means of a gas sample

\footnotetext{
${ }^{2}$ Other sensor models could be used provided they meet the required response time and measuring range as specified in GTR Number 13.
} 
loop using a gas pulser acquired from Custom Sensors Solutions (Model 1050 Gas Pulser, Oro Valley, AZ), shown in Figure 4. Sample loops are routinely used for injecting precisely known volumes of a test gas into a gas chromatograph or other analyzer. In the results described below, the test gas pulse duration was set by the ratio of the sample loop volume divided by the flow rate of the background gas. Details on the operation of a sample loop are shown in Figure 5.

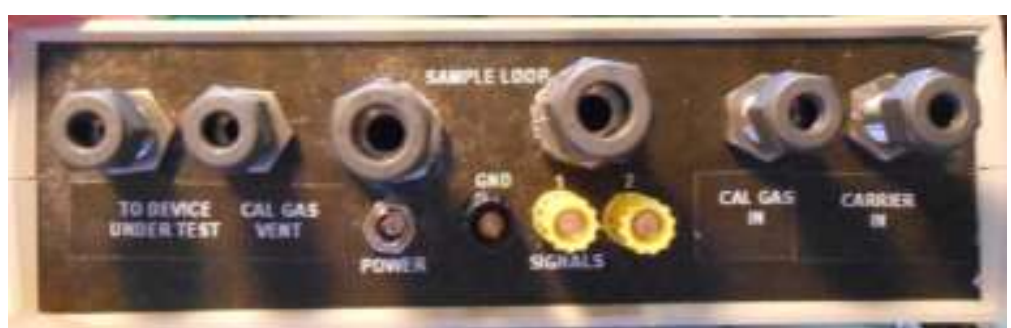

Figure 4: Front panel of the Model 1050 Gas Pulser used to generate test gas pulses of precisely known duration. (Photo used with permission)
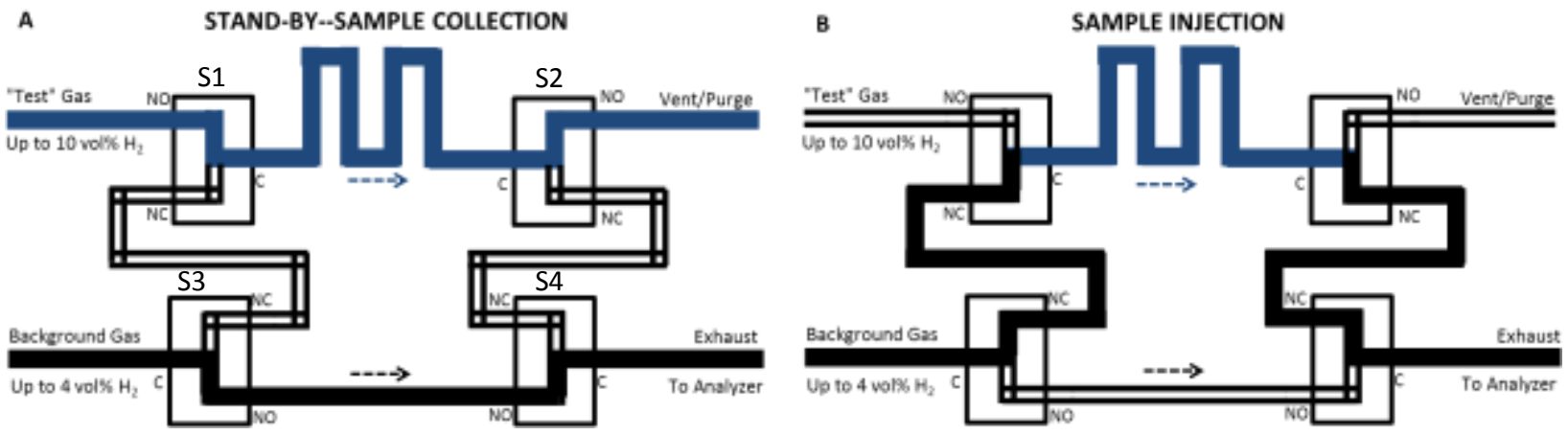

Figure 5: Gas sample loop used to control test gas concentration and gas pulse duration. Sample loops consist of two independent gas flow paths. A: One path purges the sample loop with the test gas while the second line continuously flows the background gas to the analyzer or sensor. B: Upon activation of the solenoid valves (S1 through S4), the background gas flow passes through the sample loop before reaching the sensor. The exposure time of the test gas on the sensor is the ratio of the sample loop volume to the background gas flow rate. Gas pulses of less than 1 second duration are readily achieved.

With the gas pulser, the performance of the sensor to transient gas pulses and composition could be characterized. Results to date are presented below. A variety of test procedures were performed, including determination of the sensor measuring range, the ability of the sensor to accurately measure gas exposures of less than 1 second, effect of the background gas on sensor response (e.g., air vs. nitrogen), and the impact of flow rate. Initial testing was performed with the TC sensor in the standard configuration.

Measuring Range: The TC sensor response was measured for hydrogen concentrations ranging from 0 to $2.5 \mathrm{vol} \%$ in air. For safety reasons, higher concentrations of hydrogen were in nitrogen. Accordingly, the sensor was also exposed to 0 to $10 \mathrm{vol} \% \mathrm{H}_{2}$ in nitrogen. The results are shown in Figure 6, which also indicates the actual test gas concentration. The test gas flow rate was 1 standard liter per minute (SLPM), which corresponded to a pulse width of 1.4 seconds. The sensor was subjected to six sequential hydrogen exposures for each test gas concentration. The test results indicate excellent repeatability of the sensor (see left side of Figure 6). The sensor, which was calibrated to read in vol\% $\mathrm{H}_{2}$, was also very accurate. 
The right side of Figure 6 shows an expanded view of one exposure at the indicated time window. The sensor response was logged at a rate of 1 point every $250 \mathrm{~ms}$. As indicated in Figure 6, the sensor was able to reach a stable steady-state response level within the short duration of the hydrogen pulse. Results for hydrogen in air up to $2.5 \mathrm{vol} \%$ are plotted in curves $6 \mathrm{~A}$ and $6 \mathrm{~B}$, while the results for hydrogen in nitrogen up to $10 \mathrm{vol} \%$ are plotted in curves $6 \mathrm{C}$ and $6 \mathrm{D}$. The sensor responses were found to be linear, repeatable, and fast.
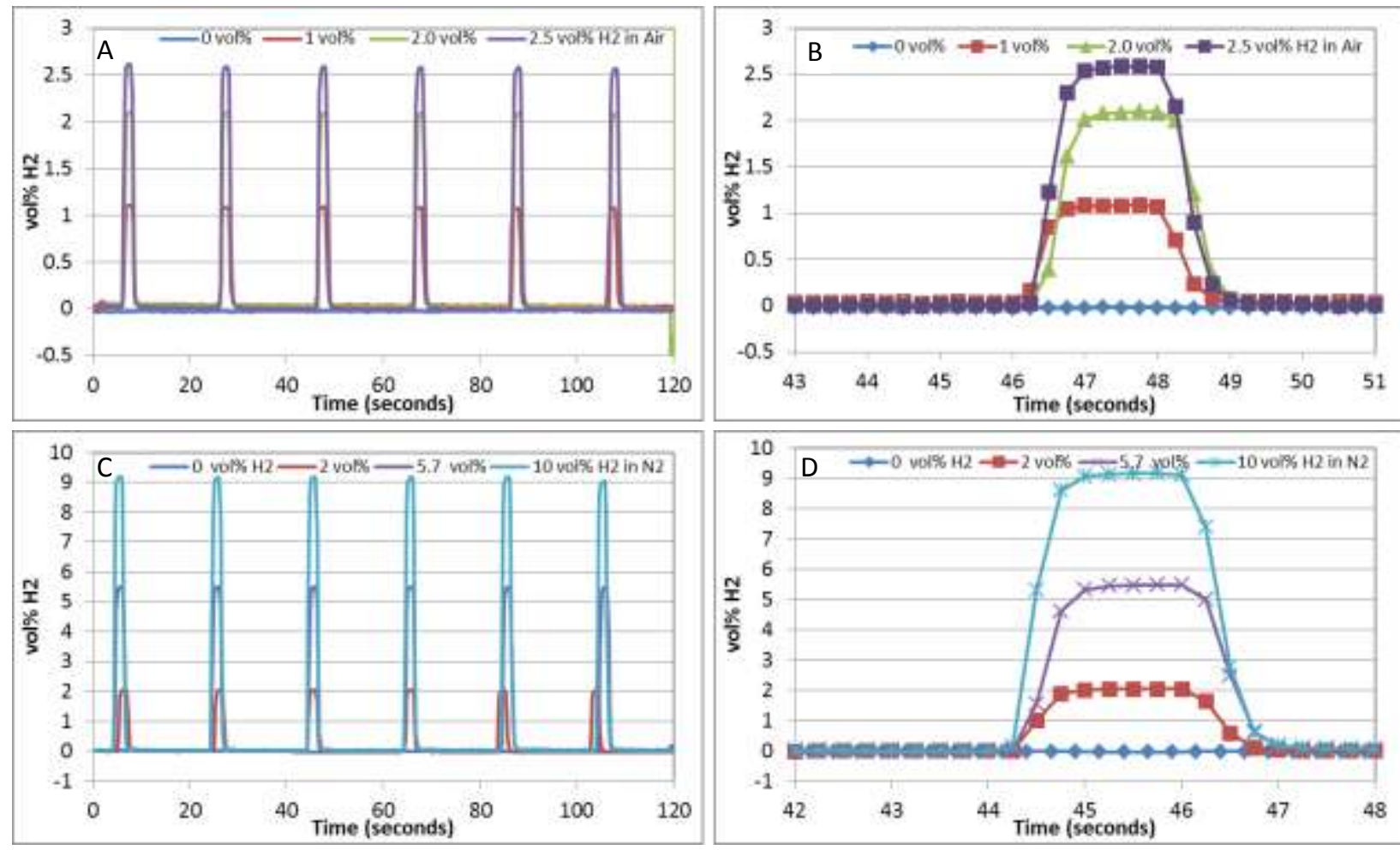

Figure 6: Response to TC sensor (normal configuration) with gas flow rates of 1 SLPM as maintained by a mass flow controller. Curves A and B were with hydrogen in air at the indicated concentration, while curves C and D were with hydrogen in nitrogen at the indicated concentration. The pulse duration was approximately $1.4 \mathrm{~s}$.

Sensor Response Time and Flow Rate Dependence: The TC sensor in the standard configuration was also evaluated at different test gas flow rates and pulse widths. Although one means to change the pulse width would be to vary the sample loop volume, in the tests described here the sample loop volume remained fixed and the flow rate was adjusted. The actual flow rate set points were $0.1,0.2,0.5,1.0,1.3,1.5$, and 2.0 SLPM, which corresponded to gas pulse widths of 14.1, 7.1, 2.8, 1.4, 1.1, 0.94, and $0.76 \mathrm{~s}$, respectively. The tests were performed in a manner identical to that shown in Figure 6 in that the sensor was subjected to six sequential exposures. Expanded data for pulse widths durations of 1.4 to $0.76 \mathrm{~s}$ are shown in Figure 7A. This clearly shows that the sensor is readily able to detect transient hydrogen transients faster than $1 \mathrm{~s}$, a critical requirement for the GTR specifications on allowable tail pipe emissions from FCEVs. The sensor indication was also shown to be independent of flow rate from 0.5 to 2.0 SLPM, but below 0.5 SLPM the sensor output tended to decrease, an indication of less heat transport from the TC sensing element to the external environment at lower flow rates.

Future Work on the FCEV Exhaust Gas Analyzer: The goal of this investigation is to develop a hydrogen vehicle exhaust analyzer to verify compliance with the GTR. The preliminary testing of the Xensor TC hydrogen sensor discussed here indicates that it meets the critical response time requirements as established in GTR Number 13. However, only a few of the metrological performance specifications of the sensor have been characterized, and further evaluations are necessary, including quantification of the 
impact of variations in temperature and humidity of the test gas on sensor response. Once this is completed, the sensor is to be configured into a field-usable analyzer compatible with the tailpipe environment. Towards this goal, the NREL sensor laboratory is working closely with the DOT to demonstrate that this technology can be used to verify compliance with the requirements of the GTR. Also, information on the development of the tailpipe analyzer is regularly shared within the SAE Fuel Cell Task Force as an avenue to communicate the findings to the FCEV original equipment manufacturers, several of which have expressed an interest in this approach for their own internal use to measure tailpipe emissions of FCEVs under various operation modes. The original equipment manufacturers have also provided feedback on the tailpipe environments.
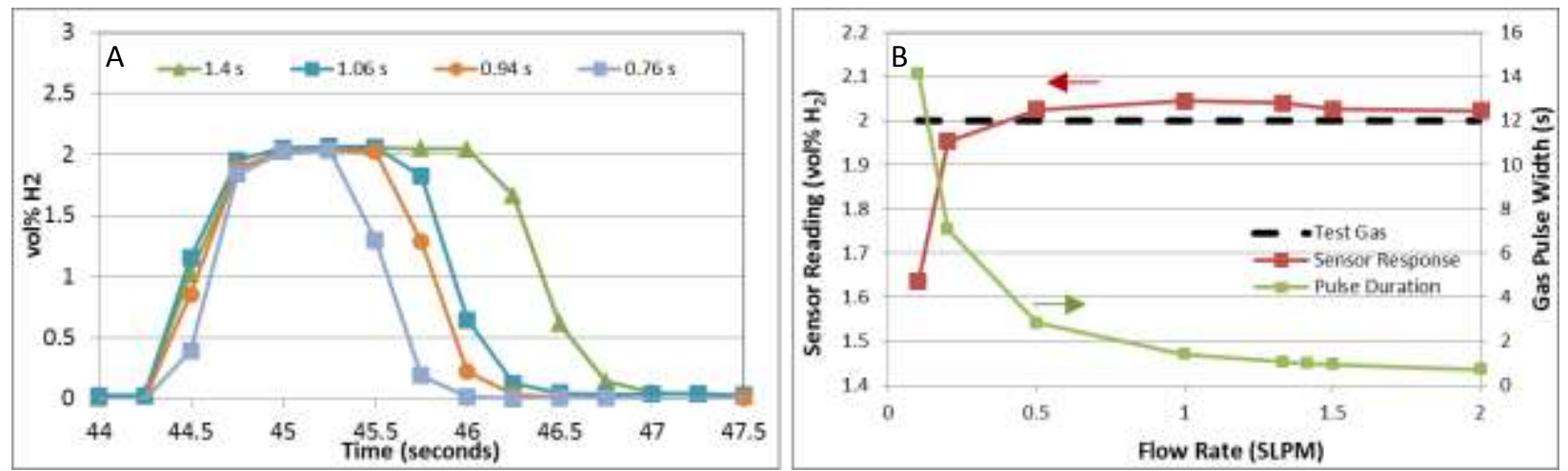

Figure 7: A: Dependence of the TC sensor in the normal configuration to respond to transient exposures of hydrogen. Pulse widths varied from 1.4 to $0.76 \mathrm{~s}$, but data shown for the expanded region are for pulse widths less than $1.4 \mathrm{~s}$. The sensor is able to accurately quantify hydrogen pulses less than $1 \mathrm{~s}$. B: A plot of the sensor response to gas flow rates and the corresponding gas pulse widths.

\section{CONCLUSIONS}

GTR Number 13 has several hydrogen monitoring requirements. Work at the NREL and JRC sensor laboratories demonstrates that commercially available sensor technology can be used to verify compliance with the GTR requirements. These findings are made available to DOT, and hence to the GTR committee and the hydrogen community. A means of verifying fuel system integrity following crash tests has been demonstrated, while the development of an FCEV exhaust gas analyzer is presently under development. A low-cost sensor for the analyzer has been identified.

\section{ACKNOWLEDGEMENTS}

The NREL Sensor Test Laboratory was supported by the Department of Energy, Hydrogen Safety Codes and Standards Program, Program Manager, Will James. The authors also acknowledge feedback and information provided by NHTSA on the relationship between the GTR and the Federal Motor Vehicle Safety Standard. DOT/NHTSA also facilitated our collaboration in the vehicle crash test. The SAE Fuel Cell Safety Task Group (Mr. Glenn Scheffler, Chair) and vehicle original equipment manufacturers are also acknowledged for providing proprietary details of the tailpipe environment and the requirements for analyzer for applications other than those prescribed in the GTR.

\section{REFERENCES}

1. DOE Fuel Cell Technologies Program, http://www1.eere.energy.gov/hydrogenandfuelcells/.

2 U.S. Department of Energy, http://energy.gov/mission.

3. http://setis.ec.europa.eu/about-setis/technology-map/2011_Technology_Map1.pdf/view. 
4. Boon-Brett, L., Bousek, J., Castello, P., Salyk, O., Harskamp, F., Aldea, L., and Tinaut, F., Reliability of Commercially Available Hydrogen Sensors for Detection of Hydrogen at Critical Concentrations: Part I - Testing Facility and Methodologies, Int J Hydrogen Energy 33, 2008, pp. 7648-7657.

5. NREL Sensor Testing Laboratory, see: http://www.nrel.gov/hydrogen/facilities hsl.html.

6. H2Sense - Hydrogen Sensors for the Safe Use of Hydrogen (http://www.h2sense.bam.de/en/home/index.htm).

7. Global Technical Regulation: Hydrogen Fueled Vehicle: Proposal to develop a global technical regulation concerning Hydrogen fuel cell vehicle (ECE/TRANS/WP.29/AC.3/17).

8. Post, M.B., Burgess, R., Rivkin, C., Buttner, W.J., O’Malley, K., and Ruiz, A., Onboard Hydrogen/Helium Sensors in Support of the Global Technical Regulation: An Assessment of Performance in Fuel Cell Electric Vehicle Crash Tests, (September 2012) National Renewable Energy Laboratory, Report NREL/TP-5600-56177. http://www.nrel.gov/docs/fy12osti/56177.pdf.

9. Buttner, W.J., Burgess, R., Rivkin, C., Post, M.B, Boon-Brett, L., Palmisano, V., and Moretto, P., An Assessment on the Quantification of Hydrogen Releases through Oxygen Displacement Using Oxygen Sensors, Int J Hydrogen Energy 39 (2014) 20484-20490.

10. Karco Engineering, http://www.karco.com/.

11. Willoughby, D.B., Royle, M., Nilsen, S., and Gautier, T., Hydrogen venting under variable flow conditions, 4th International Conference on Hydrogen Safety (ICHS), San Francisco, California, USA, 12-14 September 2011.

12. Ekoto, I.W., Merilo, E.G., Dedrick, D.E., and Groethe, M.A., Performance-Based Testing for Hydrogen Leakage into Passenger Vehicle Compartments, Int J Hydrogen Energy, 36, 2011, pp. 10169-10178.

13. Buttner, W.J., Burgess, R., Rivkin, C., Post, M.B. Boon-Brett, L., Palmisano, V. Moretto, P., An Assessment on the Quantification of Hydrogen Releases through Oxygen Displacement Using Oxygen Sensors, presented at the $5^{\text {th }}$ International Conference on Hydrogen Safety September 9-13, (2013) Brussels, Belgium.

14. Corfu, R., DeVaal, J., and Scheffler, G., "Development of Safety Criteria for Potentially Flammable Discharges from Hydrogen Fuel Cell Vehicles," (2007) SAE Technical Report 2007-01-437.

15. Hydrogen, Fuel Cells \& Infrastructure Technologies Program Multi-Year Research, Development and Demonstration Plan, Planned program activities for 2005-2015, U.S. DOE Office of Renewable Energy and Efficiency (EERE), 2005 http://www.nrel.gov/docs/fy08osti/39146.pdf

16. Boon-Brett, L., Black, G., Moretto, P., and Bousek, J., A comparison of test methods for the measurement of hydrogen sensor response and recovery times, Int J Hydrogen Energy 139 (2010) 7652-7663. 